\title{
Zinc( II ) Induced Neurological Thrombolytic Activities for COVID-19 Thrombus Prevention, Inflammation, Fibrin Degradation, Fibrinolysis of Dissolving Blood Clots and Blood Flow Reperfusion after Thrombolysis
}

\section{Tsuneo Ishida*}

Saido, Midori-Ku, Saitama-Shi, Saitama-Ken, 336-0907, Japan

"Corresponding author: Dr. Sci. Tsuneo Ishida, 2-3-6, Saido, Midori-Ku, Saitama-Shi, Saitama-Ken, 336-0907, Japan.

Citation: Ishida T (2021) Zinc( II ) Induced Neurological Thrombolytic Activities for COVID-19 Thrombus Prevention, Inflammation, Fibrin Degradation, Fibrinolysis of Dissolving Blood Clots and Blood Flow Reperfusion after Thrombolysis. Arch Surg Clin Case Rep 4: 158. DOI: 10.29011/2689-0526.100158

Received Date: 29 November, 2021; Accepted Date: 03 December, 2021; Published Date: 06 December, 2021

\section{Abstract}

Zinc( II ) induced neurological thrombolytic activities have become apparent during thrombolysis process of COVID-19 thrombus prevention, inflammation, fibrin degradation, fibrinolysis of dissolving blood clots, and blood flow reperfusion after thrombolysis. In COVID-19 thrombus prevention, zinc can prevent respiratory thrombosis and pulmonary thromboembolism by inhibition of thrombus formation growth. In COVID-19 inflammation, zinc-induced suppression of inflammation in thrombolysis may be involved that zinc can reduce the inflammation in the abdominal aortic aneurysm (AAA) which zinc has anti-inflammatory and anti-oxidant effects, and immune responses. Fibrin degradation process is involved that zinc binds tissue-type plasminogen activator (tPA), in which atorvastatin decreased cerebral, fibrin, neutrophil, and microvascular platelet deposits. $\mathrm{Zn}^{2+}$ accelerates fibrin clot formation and increases fiber diameter both in the absence and in presence of coagulation factor (FXIII) and zinc ions effect clot structure of porosity, stiffness, and rheology. In zinc-induced thrombolytic dissolving blood clots, zinc-stabilized t-PA gelatin complex, MMP9 and MMP3 with t-PA are a promising t-PA delivery system, and may facilitate neuroprotection and vital for reperfusion treatment that zinc-induced neurotoxicity has been shown to play a role in neuronal damage and death associated with traumatic brain injury, stroke, seizures, and neurodegenerative diseases with worthing a neuroprotective intervention in stroke. Furthermore, zinc induced ROS in the resolution of a venous thrombus has ROS-mediated cell recruitm and oxidative stress at the site of a venous thrombus and increased ROS production is demonstrated in during ischemia, reperfusion, and restoration of the blood flow.

Finally, the zinc( II ) binding molecular mechanism is considered that $\mathrm{Zn}^{2+}$ ions may bind to inflammatory, fibrinogen, fibrinolytic, and thrombus proteins respectively by $\mathrm{Zn}^{2+}$ ions-centered coordinated tetrahedral binding molecular pattern.

Keywords: Fibrinolysis; Zinc-stabilized tPA; Fibrin Degradation; Reperfusion after Thrombolysis; Zn-induced Neurodegenerative and Neuroprotective Intervention; $\mathrm{Zn}^{2+}$ ionscentered coordinated Molecular Binding

\section{Abbreviations}

AAA: Abdominal Aortic Aneurysm; AIS: Acute Ischemic Stroke; ARDS: Acute Respiratory Syndromes; BBB: Blood-Brain Barrier; CNS: Central Nervous System; COVID-19: Coronavitrus 
Citation: Ishida T (2021) Zinc( II ) Induced Neurological Thrombolytic Activities for COVID-19 Thrombus Prevention, Inflammation, Fibrin Degradation, Fibrinolysis of Dissolving Blood Clots and Blood Flow Reperfusion after Thrombolysis. Arch Surg Clin Case Rep 4: 158. DOI: 10.29011/2689-

Disease-19; CVDs: Cardiovascular Diseases; DECT: Dual Energy Computed Tomography; DHI: Danhong Injection; ECM: Extracellular Matrix; FDPs: Fibrin Degradation Products, FXIII F13: Coagulation Factor FXIII, ICH: Intracerebral Haemorrhage, IFN: Interferon; MMPs: Matrix Metalloproteinases; NET: Neutrophil Extracellular Trap; NO: Nitric Oxide; NOAEL: No Observed Adverse Effect Level; PE: Pulmonary Embolism; POCUS: Point-of-Care Ultrasound; PTCA: Percutaneous Transluminal Coronary Angioplasty; RNS: Reactive Nitrogen Species; ROS: Reactive Oxygen Species; RV: Right Ventricular; RVD: Right Ventricular Dysfunction; SK: Streptokinase; t-PA: tissue-Type Plasminogen Activator; TIMI: Thrombolysis in Myocardial Infarction; ZIP: Zrt, Irt-Like Protein; ZIP4: Zinc Influx Transporter4; VSMCs: Vascular Smooth Muscle Cells; VTE: Venous Thromboembolism

\section{Introduction}

Coronavirus disease-19(COVID-19) thrombolysis infectious disease has a critical therapeutic role in severe pneumonia with pulmonary embolism (PE) that point-of-care ultrasound (POCUS) has a pivotal role in the refractory acute respiratory syndrome (ARDS) in COVID-19 [1]. Rescue thrombolysis with severe COVID-19 acute respiratory syndrome (ARDS), thrombosis and right ventricular dysfunction (RVD) that has a trend to improvement in perfusion. Understandings of impact of thrombolysis on micro and macro thrombosis, ventilator efficiency and RVD with COVID-19 ARDS, the potential survival benefit, and the possible role of thrombolysis in preventing pulmonary vascular disease are required [2].

The thrombolysis procedures have been analysed from Acute Ischemic Stroke (AIS) patients with tissue-type plasminogen activator (t-PA) and the majority of treatment of system delays, and the thrombolysis treatment decision when treating patients with tPA, and study next steps including the development of a discreteevent computer simulation model to quantify the impact of these suggested changes to the current processes [3]. Treatment with tPA results in neutrophil recruitment, activation, and neutrophil extracellular trap (NET) formation that tPA has long been the single available therapeutic and nowadays, modified tPA versions and combinations with tPA have been used as a stroke therapeutic [4]. The thrombolysis using liposomes is an attractive concept toward directed dissolution of blood clots that plasmin variants are produced as direct fibrinolytic agents as a futuristic approach with targeted delivery of these drugs using liposome technology, in which its rigorous clinical testing needs to be done to understand its futuristic implications [5].

On the other hand, zinc and its protective role for therapeutic use of zinc are important as zinc bioavailability in immunity and inflammation, ARDS, pneumonia, and thrombolysis, hypoxia and ischemia reperfusion injury against COVID-19 infection [6].
Zinc( II ) induced anti-thrombosis has been investigated that zinc can inhibit inflammation, platelet behavior function, blood coagulation and promotes neurological antithrombosis formation during ROS production, excessive oxidative stress, and thrombosis process against COVID-19 infection, in which zinc ions-binding molecular mechanism has been clarified that $\mathrm{Zn}^{2+}$ ions may be bound with COVID-19 inflammatory, platelet, coagulation, thrombus proteins by $\mathrm{Zn}^{2+}$ ions-centered tetrahedral binding protein molecules coordination pattern [7]. Thrombolysis in COVID-19 infection is involved that zinc( II) induced thrombolysis is proceeded by zinc concentration from 0.5 to $1 \mu \mathrm{M}$, in which the molecular mechanism is clarified that $\mathrm{Zn}^{2+}$ ions could bind with COVID-19 RNA thrombolytic proteins by $\mathrm{Zn}^{2+}$-centered tetrahedral coordination pattern [8]. Zinc serves neurological importance to the function of the central nervous system (CNS) that zinc induced neuroprotection with specificity against zinc-mediated injury, tissue plasminogen activator (tPA), tPA's biological effect, and neurodegenerative disease for thrombolysis have increasingly important roles for thrombolysis in human patients [9].

Zinc induced productive reactive oxygen species (ROS) play a role in the reperfusion injury that enhances ROS scavenging and/or detoxification protect against reperfusion injury, artificial generation of ROS in otherwise normal tissue recapitulating the injury response, and detection of enhanced ROS production, in which meaningful progress as ROS-directed drugs that can be used to effectively treat reperfusion injury [10].

In this semi-review, thrombolysis process may be assumed to be consisted of COVID-19 thrombus prevention, inflammation, fibrin degradation, dissolving blood clots, and blood flow, reactive oxygen species (ROS) generation, and reperfusion after thrombolysis, in which zinc( II ) induced neurological thrombolytic activities during thrombolysis process against COVID-19 infection are revealed, subsequently, the zinc binding molecular mechanism has been clalified.

\section{Thrombolysis process}

Physical and mechanistic features of thrombolysis have a bolus of tPA at the clot edge, the effects of clot structure on clot degradation, plasminogen molecules, and the effects of modifcations to fibrinolytic drugs or dosing regimens, in which these facts have signifcant implications for thrombolytic treatment in fibiolysis at the clot or thrombus edge, fibriolysis proceeding, degradation of fbrin and tPA release, fibriolysis proceeding, and effects of modifcations to fibrinolytic drugs [11].

Thrombolysis refers to fibrinolysis and dissolution of the thrombus that fibrinolysis acts by activation of plasminogen to fibrinogen and fibrin and lyses the clot, which then allows reperfusion of the ischaemic brain. Thrombolytic agents include streptokinase 
Citation: Ishida T (2021) Zinc( II ) Induced Neurological Thrombolytic Activities for COVID-19 Thrombus Prevention, Inflammation, Fibrin Degradation, Fibrinolysis of Dissolving Blood Clots and Blood Flow Reperfusion after Thrombolysis. Arch Surg Clin Case Rep 4: 158. DOI: 10.29011/2689-

(SK) and recombinant tissue-type plasminogen activator (rt-PA). SK has a longer halflife than tissue-type plasminogen activator (tPA), prevents re-occlusion and is degraded enzymatically in the circulation. rt-PA is more fibrin-specific and clot-dissolving, and is metabolized during the first passage in the liver [12].

Thrombolysis process with fibrinolysis is considered that fibrinolysis refers specifically to the agents causing fibrin breakdown in the clot, namely antifibrinolytics, such as aminocaproic acid ( $\varepsilon$-aminocaproic acid) and tranexamic acid are used as inhibitors of fibrinolysis. Accordingly, the thrombolysis process may consist of thrombosis prevention, t-PA and zinc agents, anti-inflammatory agent, fibrinolytic system, degradation, dissolving blood clots, and blood flowing stability after thrombolysis. Zinc-tPA connection promotes thrombolysis process, however the thrombolysis process in dissolution of the thrombus remains poorly understood yet, but this study is investigated on the thrombolytic activity during thrombolysis process. Hence, zinc induced thrombolystic activities can be obvious during thrombolysis process of (1)COVID-19 thrombus prevention, (2)Inflammation, (3)Fibrin degradation, (4)Fibrino- lysis of dissolving blood clots, and (5)Blood flowing stability with t-PA using, ROS generation, and reperfusion after thrombolysis as given below.

\section{Zinc promotes COVID-19 thrombus prevention}

Intracellular zinc homeostasis is beneficial for prevention of cardiovascular diseases (CVDs) that zinc tranporters can prevrent endothelial CVDs and vascular smooth muscle cells (VSMCs) functions [13]. For prevention of SARS-CoV-2 and most importantly for general health, given that zinc supplements are readily available, they may be indicated for people with low or borderline low results, low dietary intake and/or increased needs. To optimise safety, a daily dose lower than the tolerable upper limits ( $<7 \mathrm{mg}$ for children aged 1-3 years up to $22 \mathrm{mg}$ for those aged 15-17 years) should be used along with dietary modifications whenever possible. In adults, doses up to the no observed adverse effect level (NOAEL) of $50 \mathrm{mg} /$ day should be considered [14]. Zinc serves for thrombus prevention as a ubiquitous modulator of haemostasis and thrombosis, aggregation, anti-coagulation and fibrinolysis that zinc controls blood clot formation on fibrin(ogen) expression and function, in which demonstrate its central role in clot formation during hemostasis and thrombosis [15]. Zinc can prevent respiratory thrombosis and pulmonary thromboembolism by inhibition of thrombus formation growth in COVID-19 infection [16].

\section{Zinc inhibits COVID-19 inflammation in thrombolysis}

Inflammation increases procoagulant factors, and also inhibits natural anticoagulant pathways and fibrinolytic activity, causing a thrombotic tendency [17]. Inflammation of venous wall in thrombosis is usually induced by vessel wall damage, stimulates the coagulation system that coagulation factors inhibit the release of anticoagulant factors and hamper endogenous fibrinolysis. As thrombus formation is based on the activation of coagulation system, provoked by inflammation prevention and treatment of venous thromboembolism (VTE), should include both anticoagulant and anti-inflammatory agents [18]. Inflammatory responses to intracerebral hemorrhage (ICH) are quantified molecular markers of inflammation in human brain tissue, negative regulation of apoptosis and neutrophil degranulation, and neuroinflammatory response after $\mathrm{ICH}[19]$.

Zinc-induced suppression of inflammation in thrombolysis may be involved that zinc can reduce the inflammation in the abdominal aortic aneurysm (AAA) and successful thrombolysis is related to a significantly attenuated inflammatory response [20,21]. Zinc has anti-inflammatory and anti-oxidant effects, and immune responses by cytokine-activated macrophages, which release NO in high concentrations that zinc deficiency affects the generation of inflammatory cytokines, in which these zinc status-involved inflammatory responses often commingle with their antioxidant deficiency induced-oxidative stress results in the reduction of NO in epithelium [22].

\section{Zinc induced COVID-19 fibrin degradation and fibrinolysis controls blood coagulation}

Fibrin/fibrinogen degradation products and D-dimer have been positively correlated with injury severity score, and the relationships varied according to trauma severity [23]. Fibrinolysis controls blood coagulation that hypofibrinolysis resulting in impaired clot dissolution is caused to thrombosis in multiple disease states that the mechanism of the anticoagulant effect of fibrin degradation products (FDPs) is unlikely that FDPs exert any target-specific feedback inhibition or amplification of thrombosis [24].

$\mathrm{Zn}^{2+}$ has competing effects on clot formation and degradation via distinct mechanisms that zinc delays clot lysis and attenuates fibrin degradation that zinc accelerates clotting and reduces fibrin clot stiffness in a coagulation factor F13-independent manner, suggesting that zinc may work in concert with F13 (FXIII) to modulate clot strength and stability [25]. $\mathrm{Zn}^{2+}$ slows fibrinolysis by attenuating plasminogen activation and fibrin degradation by plasmin and zinc circulates free in plasma at a concentration of $0.1-2 \mu \mathrm{M}$, In $5 \mu \mathrm{M}$ zinc presence, the catalytic efficiency of plasminogen activation by tPA was reduced by approximately two-fold. Zinc had no effect on the affinity of plasminogen or plasmin for fibrin, but increased the affinity of tPA by two-folds. $\mathrm{Zn}^{2+}$ serves as a dynamic modulator of numerous reactions in haemostasis, and contributes to the localized, rather than systemic, response to perturbation, in which zinc modulates fibrinolysis by attenuating tPA-mediated plasminogen activation and plasmininduced fibrin degradation [26]. The tPA-induced upregulation of matrix metalloproteinase-9 (MMP-9) is reduced by atorvastatin, 
Citation: Ishida T (2021) Zinc( II ) Induced Neurological Thrombolytic Activities for COVID-19 Thrombus Prevention, Inflammation, Fibrin Degradation, Fibrinolysis of Dissolving Blood Clots and Blood Flow Reperfusion after Thrombolysis. Arch Surg Clin Case Rep 4: 158. DOI: 10.29011/2689-

in which atorvastatin decreased cerebral, fibrin, neutrophil, and microvascular platelet deposits that atorvastatin-mediated neuroprotective effects are responsible for the reduction of adverse effects resulting from delayed tPA treatment [27].

Fibrinogen is the immediate precursor of fibrin blood clots that $\mathrm{Zn}^{2+}$ in alkaline phosphatase tracer (ZnTAP) binds to fibrinogen, in which the results support a role for protein conformation in $\mathrm{Zn}^{2+}$ binding and demonstrate the utility of the ZnTAP complex with tracer for fibrinogen binding interactions [28].

The role of ROS in the resolution of venous thrombus has ROS-mediated cell and oxidative stress at the site of a venous thrombus. The recruited cells promote thrombus resolution through phagocytic action removing red cell mass, thrombus and vein wall remodeling including fibrin degradation and the stimulation of extracellular matrix (ECM) deposition and neovessel formation [29].

\section{Zinc induced thrombolytic dissolving blood clots}

Fibrinolysis in COVID-19 ARDS is involved in the impact of thrombolysis on micro- and macrothrombosis, ventilatory efficiency, and RVD in these complex patients with COVID-19 ARDS [2]. Danhong injection (DHI) could inhibit the activation of MMP-9 and protect the blood-brain barrier (BBB), which may reduce the MMP-9 activation caused by t-PA and the destruction of the BBB. DHI $(4 \mathrm{~mL} / \mathrm{kg})$ combined with t-PA $(2.5 \mathrm{mg} / \mathrm{kg})$ may reduce ischemic brain damage, intracerebral haemorrhage (ICH) and blood brain barrier damage, in which this combination may be ultimately facilitate new therapeutic approaches to enhance t-PA thrombolysis in stroke patients [30]. Zinc binds fibrin(ogen) and attenuates fibrinolysis that $\mathrm{Zn}^{2+}$ accelerates fibrin clot formation and increases fibre diameter both in the absence and presence of coagulation factor (FXIII). Zinc ions effect clot structure of porosity, stiffness, and rheology that $\mathrm{Zn}^{2+}$ serves as a dynamic modulator of numerous reactions in haemostasis and contributes to the localized, rather than systemic, response to perturbation, and that ionic conditions should be considered when evaluating the effects of FXIIIa on clot [24]. $\mathrm{Zn}^{2+}$ binds to fibrinogen with high affinity and these fibrin bundles are loosely coupled from a mechanical perspective, possibly resulting from the flexibility of the alpha-C regions of fibrinogen that excess $\mathrm{Zn}^{2+}$ in the microenvironment within the clot can be used in tissue engineering applications to design hierarchical fibrin scaffolds with multiscale control over the mechanical properties. Thus, the mechanics of a fibrin network are closely correlated with its microscopic structure and inform our understanding of the structure and physical mechanisms leading to defective or excessive clot stiffness [31].

Zinc induced blood flow, zinc with t-PA using in fibrinolysis, ROS and RNS gneration, and reperfusion after thrombolysis.

Coronary blood flow after thrombolysis is flow of percutaneous transluminal coronary angioplasty (PTCA) of the residual stenosis, delayed nonculprit artery, and following relief of the stenosis by stenting that increased residual stenosis severity is associated with thrombolysis in myocardial infarction (TIMI) flow grade frame counts [32]. The release of ionic $\mathrm{Zn}^{2+}$ store from secretory granules upon platelet activation contributes to the procoagulant role of $\mathrm{Zn}^{2+}$ in platelet-dependent fibrin formation [33].

The rationale for tPA treatment for COVID-19 ARDS is by the need for effective fibrinolysis. The systemic tPA in patients with severe COVID-19 and ARDS has been clinically suspected on pulmonary thrombosis that COVID-19 thrombolysis significantly improved ventilatory parameters as well as a trend to echocardiographic measures of right ventricular (RV) function that thrombolysis led to a sustained trend to improvement on the follow-up dual energy computed tomography (DECT) scans available, in terms of micro and macrovascular perfusion [2].

Zinc stabilized tPA gelatin is that The zinc-stabilized t-PA gelatin complex is a promising t-PA delivery system which can manipulate the thrombolytic activity by the local ultrasound irradiation [34]. Zinc-bound-tPA interacts with the zinc influx transporter Zrt, Irt-like Protein (ZIP) ZIP4 through the kringle domain of tPA with the extracellular region of the transporter facilitating zinc and ZIP4 vesicular internalization that tPA facilitates the partial sequestration of zinc into lysosomal compartments via ZIP4, thereby regulating the intracellular concentrations of zinc and acting in a neuroprotective manner by modulating intracellular signaling events that could lead to cell death [35].

Matrix metalloproteinases (MMPs) comprising a family of at least 25 zinc-dependent endopeptidases have been shown to display critical activities throughout the repair phases of cerebral ischemia, particularly during angiogenesis and reestablishment of cerebral blood flow. MMP10 reverses tPA-induced excitotoxicity in cortical neurons that MMP10, either alone or in combination with tPA, might provide for higher protection against cerebrovascular damage and thrombolysis [36]. MMP-9 plays a central role in tPA-mediated neurotoxicity from thrombolytic therapy for acute stroke that Activated MMP-9 degrades the neurovascular matrix, and tPA amplifies total levels of MMP-9 after ischemia. Perfusion of tPA resulted in BBB disruption and degradation of the basal membrane protein. Treatment of MMP9 and MMP3 with t-PA also may facilitate neuro-protection and hasten rescue of brain tissue following stroke, vital for reperfusion treatment, improving potential clinical benefits of a combined therapy [37].

$\mathrm{Zn}^{2+}$ toxicity plays an important role in ischemic neuronal death that $\mathrm{Zn}^{2+}$ chelators have the potential to be used in the patients treated with intravenous thrombolysis (within $3 \mathrm{~h}$ of ischemic stroke onset) as an adjunctive therapy treated with mechanical thrombectomy through intra-arterial delivery to prevent the 
Citation: Ishida T (2021) Zinc( II ) Induced Neurological Thrombolytic Activities for COVID-19 Thrombus Prevention, Inflammation, Fibrin Degradation, Fibrinolysis of Dissolving Blood Clots and Blood Flow Reperfusion after Thrombolysis. Arch Surg Clin Case Rep 4: 158. DOI: 10.29011/2689-

reperfusion injury with worthing a neuroprotective intervention in stroke [38]. Zinc-induced neurotoxicity has been shown to play a role in neuronal damage and death associated with traumatic brain injury, stroke, seizures, and neurodegenerative diseases that MMP-9 may underlie tPA-induced disruption of the blood brain barrier $(\mathrm{BBB})$ and the addition of neuroprotective agents might come about through the understanding of the toxicity of tPA and the invention of agents that will block the toxicity without affecting its ability to thrombolyse [39].

Reperfusion after thrombolysis may be explained by downstream displacement of clot material and microembolism of the vascular periphery. Incomplete tissue recovery despite sufficient reperfusion, which might be due to effects previously associated with reperfusion injury. Further understanding of the pathophysiologic mechanisms during and early after thrombolysis may have an impact on treatment strategies in stroke patients [40]. Limiting reperfusion damage and recovering from ischemic stroke are stem cell modification that the disadvantages of IV transplantation can be solved, resulting in IV transplantation being the most prominent in the future, especially in acute ischemic stroke. However, chronic stroke is more challenging with IV transplantation, as chemotactic signaling is weaker in the chronic stage. Therefore, intracranial transplantation may be required in patients with chronic stroke [41].

$\operatorname{Zinc}\left(\mathrm{ZnCl}_{2}=10,50,100 \mu \mathrm{M}\right)$ inhibited streptokinaseinduced blood clot thrombolysis and treatment with zinc chelator promotes streptokinase-induced thrombolysis. This utilization of a proper dose of streptokinase could improve reperfusion in the treatment of thrombotic disorders [42].

The other, zinc induced ROS and RNS generation play an important role for reperfusion in thrombolysis (fibrinolysis) that free radicals, reactive oxygen species (ROS) and reactive nitrogen species (RNS), have intense oxidation or nitrification abilities that during cerebral ischemia reperfusion, especially with blood reflow, massive generation of ROS and RNS leads to cell death via DNA damage, protein dysfunction, and lipid peroxidization. Hence, oxidative/nitrosative stress in ischemia-reperfusion injury also plays a key role in inducing hemorrhagic transformation and cerebral edema after revascularization therapy [43]. Inhibition of ROS production may reduce autophagy to suppress platelet activation of oxidized low-density lipoprotein by activating the mTOR signal pathway [44]. ROS can modulate the cytokineinduced tPA for ROS mainly derived from neutrophils and antiproteases that NO and ROS have opposing effects on tPA [45]. Increased ROS production is demonstrated in both during ischemia and reperfusion, and restoration of the blood flow can save brain tissue. However, reperfusion might have some deleterious effects that although oxidative stress biomarkers related to lipid peroxidation are associated with stroke severity and outcome, no identifiable changes in their concentrations are observed after t-PA-induced recanalization [46]. The accumulation of ROS contributing to reperfusion injury is supported by a large and growing body of evidence that the presence of risk factors has revealed a more robust production of ROS and greater tissue damage following reperfusion, and meaningful progress can be made towards the translation of preclinical findings to the clinical setting [10].

In addition, the temporal relationship between NO and ROS generation may be a critical step in the modulation of tPA and PAI-1 expression in mesangial cells and may account for a DE dysregulation of matrix turnover during inflammatory processes in the renal mesangial that ROS scavengers in a combination regimen may play a crucial role with acute ICH [47], and treatment with tPA results in neutrophil recruitment, activation, and neutrophil extracellular trap (NET) formation that provokes the breakdown of the blood-brain barrier (BBB) and intracerebral hemorrhage (ICH) through a type I interferon (IFN) response in the ischemic brain [48].

Thus, zinc with t-PA, zinc-bound-tPA, zinc stabilized tPA gelatin, MMPs, streptokinase-induced zinc chelator, and zincinduced generation ROS play important roles in neuronal damage, reperfusion injury, and improving reperfusion after thrombolysis against revascularization therapy.

As mentioned above, zinc( II ) induced neurological thrombolytic activities during thrombolysis process of COVID-19 thrombus prevention, inflammation, fibrin degradation, fibrinolysis of dissolving blood clots, and blood flow stability, ROS generation and reperfusion after thrombolysis are shown in (Table 1). 
Citation: Ishida T (2021) Zinc( II ) Induced Neurological Thrombolytic Activities for COVID-19 Thrombus Prevention, Inflammation, Fibrin Degradation, Fibrinolysis of Dissolving Blood Clots and Blood Flow Reperfusion after Thrombolysis. Arch Surg Clin Case Rep 4: 158. DOI: 10.29011/2689-

\begin{tabular}{|c|c|c|c|c|c|}
\hline $\begin{array}{l}\mathrm{Zn}^{2+} \\
\text { ions }\end{array}$ & \multicolumn{5}{|c|}{$\begin{array}{l}\text { Zinc induced COVID-19 neurological thrombolytic activities for (1)Thrombosis prevention, } \\
\text { (2)Inflammation, (3)Fibrin degradation, (4)Fibrinolysis of dissolving blood clots, and } \\
\text { (5)Blood flow stability, ROS generation, and reperfusion after thrombolysis. }\end{array}$} \\
\hline \multirow{3}{*}{$\underset{\mathbf{Z n}}{\rightarrow+}$} & $\begin{array}{l}\text { Thrombosis } \\
\text { prevention }\end{array}$ & Inflammation & Fibrin degradation & $\begin{array}{l}\text { Fibrinolysis of } \\
\text { dissolving blood } \\
\text { clots }\end{array}$ & $\begin{array}{l}\text { Blood flowing } \\
\text { stability, reperfusion } \\
\text { after thrombolysis }\end{array}$ \\
\hline & $\begin{array}{l}\rightarrow \mathbf{Z n}^{2+} \text {, } \\
\text { - } \mathrm{n}^{2+} \text {-induced } \\
\text { platelet activation } \\
\text { enhances anti- } \\
\text { thrombus growth. } \\
\text { - ROS resolve } \\
\text { venous thrombus } \\
\text { - Zinc promotes } \\
\text { COVID- } 19 \\
\text { reduced neurolo- } \\
\text { gical anti- } \\
\text { thrombosis and } \\
\text { anti-ischemics }\end{array}$ & $\begin{array}{l}\longrightarrow \mathbf{Z n}^{2+} \text {, NO } \\
\text {-Anti-inflammatory, } \\
\text { anti-oxidant } \\
\text { effects, NO in } \\
\text { epithelium } \\
\text { - Zinc can reduce } \\
\text { inflammation in } \\
\text { AAA and attenuate } \\
\text { inflammatory } \\
\text { response in } \\
\text { successful } \\
\text { thrombolysis }\end{array}$ & $\begin{array}{l}\longrightarrow \mathbf{Z n}^{2+}, \mathbf{t}-\mathbf{P A}, \mathbf{R O S} \\
\text {-Zinc attenuates } \\
\text { plasmin-induced } \\
\text { fibrin degradation } \\
\text { - Zinc(II) chelate } \\
\text { binds to fibrinogen } \\
\text { - ZnTAP binds to } \\
\text { fibrinogen for } \\
\text { protein conformation } \\
\text { - ROS-mediated } \\
\text { recruited cells } \\
\text { promote thrombus } \\
\text { resolution including } \\
\text { fibrin degradation }\end{array}$ & $\begin{array}{l}\rightarrow \mathbf{Z n}^{2+}, \mathbf{t}-\mathbf{P A} \\
\cdot \text { Zinc promotes } \\
\text { fibriolysis of } \\
\text { dissolving blood } \\
\text { clots } \\
\cdot \mathrm{Zn}^{2+} \text { attenuates } \\
\text { fibrinolysis } \\
\text { - Zinc chelator } \\
\text { promotes } \\
\text { streptokinase- } \\
\text { induced } \\
\text { thrombolysis }\end{array}$ & $\begin{array}{l}\rightarrow \mathbf{Z n}^{2+} \text {, t-PA, ROS } \\
\text {-Zinc chelation } \\
\text { streptokinase- } \\
\text { induced improved } \\
\text { thrombolysis } \\
\cdot \mathrm{Zn}^{2+} \text {-tPA combination } \\
\text { promotes a stable } \\
\text { thrombolysis } \\
\cdot \text { MMPs or combinated } \\
\text { with t-PA promote } \\
\text { thrombolysis } \\
\text { - Zinc-stabilized t-PA } \\
\text { gelatin complex } \\
\cdot \text { Zn } \\
\text { - ROS production } \\
\text { and accumulation } \\
\text { demonstrated in } \\
\text { reperfusion injury }\end{array}$ \\
\hline & \multicolumn{5}{|c|}{$\begin{array}{l}\text { Zinc binding molecular mechanism is involved that } \mathrm{Zn}^{2+} \text { may bind to inflammatory, fibrinogen, fibrinolytic, an } \\
\text { thrombolytic proteins, respectively, by } \mathrm{Zn}^{2+} \text { ions-centered coordinated tetrahedrally binding pattern }\end{array}$} \\
\hline
\end{tabular}

Table 1: Zinc( II ) induced neurological thrombolytic activities during thrombolysis process of thrombus. Prevention, inflammation, fibrin degradation, fibrinolysis of dissolving blood clots, and blood Flow stability, ROS generation and reperfusion after thrombolysis against COVID-19 infection.

Finally, $\mathrm{Zn}^{2+}$ ions-protein coordinated binding model is involved that the interaction had been found on the binding specificity by $\mathrm{Zn}^{2+}$ ions-centered tetrahedral geometric coordination of the inhibitors against $3 \mathrm{C}$ proteases. Zinc ions complexes as SARS-CoV-2 3C-like protease inhibitors may play important role for this $\mathrm{Zn}^{2+}$-centered coordination pattern that the zinc-coordinating inhibitor of tetrahedral zinc sites is tetrahedrally coordinated binding to such as the catalytic triad of Serine, Histidine and Aspartate Hydrogen Residues [49].
Accordingly, zinc( II ) ions molecular binding mechanism is involved in respiratory and pulmonary organ, anti-thrombus formation, and ZAP-mediated RNA replication that zinc ions may be bound with respiratory and pulmonary proteins, thrombosis proteins, and $\mathrm{Zn}^{2+}$-RNA gene reaction. Hence, the zinc( II ) binding molecular mechanism is considered that $\mathrm{Zn}^{2+}$ ions may bind to inflammatory, fibrinogen, fibrinolytic, and thrombolysis proteins, respectively, by $\mathrm{Zn}^{2+}$ ions-centered coordinated tetrahedrally binding molecular pattern [50]. 
Citation: Ishida T (2021) Zinc( II ) Induced Neurological Thrombolytic Activities for COVID-19 Thrombus Prevention, Inflammation, Fibrin Degradation, Fibrinolysis of Dissolving Blood Clots and Blood Flow Reperfusion after Thrombolysis. Arch Surg Clin Case Rep 4: 158. DOI: 10.29011/2689-

\section{Conclusions}

Zinc(II) induced neurological thrombolytic activities during thrombolysis process of COVID-19 thrombus prevention, inflammation, fibrin degradation, fibrinolysis of dissolving blood clots, and blood flow, ROS generation and reperfusion after thrombolysis have been discussed, subsequently the zinc binding molecular mechanism is clarified as given below.

\section{Zinc induced COVID-19 thrombus prevention}

Intracellular zinc homeostasis and zinc tranporters are beneficial for prevention of CVDs and VSMCs functions. Zinc serves for thrombus prevention as a ubiquitous modulator of haemostasis and thrombosis, aggregation, anti-coagulation and fibrinolysis that zinc controls blood clot formation on fibrin(ogen) expression and function, in which demonstrate its central role in clot formation during hemostasis and thrombosis Zinc can prevent respiratory thrombosis and pulmonary thromboembolism by inhibition of thrombus formation growth in COVID-19 infection.

\section{Zinc induced inflammatory thrombolysis}

Zinc-induced suppression of inflammation in thrombolysis may be involved that zinc can reduce the inflammation in the AAA and successful thrombolysis is related to a significantly attenuated inflammatory response. Zinc has anti-inflammatory and anti-oxidant effects, which release NO in high concentrations, in which these zinc status-involved inflammatory responses often commingle with their antioxidant deficiency induced-oxidative stress results in the reduction of NO in epithelium.

\section{Zinc induced fibrin degradation and fibrinolytic blood coagulation}

Zinc delays clot lysis and attenuates fibrin degradation that zinc accelerates clotting and reduces fibrin clot stiffness in a coagulation factor F13-independent manner, suggesting that zinc may modulate clot strength and stability. Fibrinolysis controls blood coagulation that hypo fibrinolysis resulting in impaired clot dissolution is caused to thrombosis in multiple disease states.

Zinc had no effect on the affinity of plasminogen or plasmin for fibrin, but zinc-tPA binding has been increased the affinity of tPA by two-fold. The tPA-induced upregulation of MMP-9 is reduced by atorvastatin, in which atorvastatin decreased cerebral, fibrin, neutrophil, and microvascular platelet deposits that atorvastatinmediated neuroprotective effects are responsible for the reduction of adverse effects resulting from delayed tPA treatment.

\section{Zinc induced thrombolytic dissolving blood clots.}

$\mathrm{Zn}^{2+}$ accelerates fibrin clot formation and increases fibre diameter in both the absence and presence of coagulation factor (FXIII), that zinc ions effect clot structure of porosity, stiffness, and rheology. $\mathrm{Zn}^{2+}$ serves as a dynamic modulator of numerous reactions in haemostasis, and contributes to the localized, rather than systemic, response to perturbation.

DHI $(4 \mathrm{~mL} / \mathrm{kg})$ plus t-PA $(2.5 \mathrm{mg} / \mathrm{kg})$ could extend the t-PA treatment time windows to $4.5 \mathrm{~h}$, ameliorate $\mathrm{BBB}$ disruption, reduce infarction, brain swelling and hemorrhage after ischemic stroke that DHI could inhibit the activation of MMP-9 and protect the BBB, which may reduce the MMP-9 activation caused by t-PA and the destruction of the BBB.

Zinc inhibited streptokinase-induced blood clot thrombolysis and treatment with zinc chelator promotes streptokinase-induced thrombolysis.

\section{Blood flow, zinc with t-PA in fibrinolysis, ROS generation, and reperfusion after thrombolysis}

Zinc-stabilized t-PA gelatin complex is a promising t-PA delivery system, which can manipulate the thrombolytic activity. Zinc-bound-tPA interacts with the ZIP4 through the kringle domain of tPA with the zinc extracellular region.

MMPs have been shown to display critical activities throughout the repair phases of cerebral ischemia, particularly during angiogenesis and reestablishment of cerebral blood flow. MMP10 with tPA might provide for higher protection against cerebro-vascular damage and thrombolysis and MMP-9 plays a central role in tPA-mediated neurotoxicity from thrombolytic therapy for acute stroke. Treatment of MMP9 and MMP3 with t-PA also may facilitate neuro-protection and hasten rescue of brain tissue following stroke, vital for reperfusion treatment, improving potential clinical benefits of a combined therapy.

Zinc-induced neurotoxicity has been shown to play a role in neuronal damage and death associated with traumatic brain injury, stroke, seizures, and neurodegenerative diseases. $\mathrm{Zn}^{2+}$ chelators have the potential to be used in the patients treated with intravenous thrombolysis (within $3 \mathrm{~h}$ of ischemic stroke onset), to prevent the reperfusion injury with worthing a neuroprotective intervention in stroke.

Zinc induced free radicals, ROS and RNS generation, have intense oxidation or nitrification abilities that during cerebral ischemia reperfusion, especially with blood reflow, massive generation of ROS and RNS leads to cell death via DNA damage, protein dysfunction, and lipid peroxidization. Hence, oxidative/ nitrosative stress in ischemia-reperfusion injury also plays a key role in inducing hemorrhagic transformation and cerebral edema after revascularization therapy.

The role of ROS in the resolution of a venous thrombus has ROS-mediated cell recruitm and oxidative stress at the site of a venous thrombus. Increased ROS production is demonstrated in both during ischemia and reperfusion, and restoration of the blood flow can save brain tissue and the accumulation of ROS contributing 
Citation: Ishida T (2021) Zinc( II ) Induced Neurological Thrombolytic Activities for COVID-19 Thrombus Prevention, Inflammation, Fibrin Degradation, Fibrinolysis of Dissolving Blood Clots and Blood Flow Reperfusion after Thrombolysis. Arch Surg Clin Case Rep 4: 158. DOI: 10.29011/2689-

to reperfusion injury has revealed a more robust production of ROS and greater tissue damage following reperfusion, novel ROSdirected drugs can be used to effectively treat reperfusion injury.

Thus, zinc with t-PA, zinc-bound-tPA, zinc stabilized tPA gelatin, MMPs, streptokinase-induced zinc chelator, and zincinduced generation ROS play important roles in neuronal damage, reperfusion injury, and improving reperfusion after thrombolysis against revascularization therapy.

Finally, zinc( II )-protein binding molecular mechanism is considered that $\mathrm{Zn}^{2+}$ ions may bind to inflammatory, fibrinogen, fibrinolytic, and thrombolytic proteins, respectively, by $\mathrm{Zn}^{2+}$ ionscentered coordinated tetrahedrally binding molecular pattern.

Conflicts of Interest: The author declares there is no conflicts of interest.

Sources of funding: None, author's own expenses.

\section{References}

1. Abdulrahman Alharthy, Fahad Faqihi, John Papanikolaou, Abdullah Balhamar, Mike Blaivas, et al (2021); Thrombolysis in severe COVID-19 pneumonia with massive pulmonary embolism. American Journal of Emergency Medicine 41: 1-4.

2. Laura C. Price, Benjamin Garfield, Caroline Bleakley, Archie G.M. Keeling, Charles Mcfadyen, et al (2020); Rescue therapy with thrombolysis in patients with severe COVID-19-associated acute respiratory distress syndrome Pulmonary Circulation. 10: 1-5.

3. Tessa Bulmer, David Volders, and Noreen Kamal (2021); Analysis of Thrombolysis Process for Acute Ischemic Stroke in Urban and Rural Hospitals in Nova Scotia Canada. Frontiers in Neurology. 12: 1-15.

4. Iordanis Gravanis and Stella E. Tsirka (2008); tPA as a therapeutic target in stroke Expert Opin Ther Targets. 12: 1-18.

5. Adivitiya and Yogender Pal Khasa (2017); The evolution of recombinant thrombolytics: Current status and future directions. Bioengineered 8: 331-358.

6. Vidyasagar Chinni, John El-Khoury, Marlon Perera, Rinaldo Bellomo, Daryl Jones, et al (2021); Zinc supplementation as an adjunct therapy for COVID-19: Challenges and opportunities Br J Clin Pharmacol. 19: $1-10$.

7. T. Ishida (2021); Zinc( II )-induced neurological anti-thrombus formation against severe COVID-19 infection Aditum Journal of Clinical and Biomedical Research 2:1-8.

8. T. Ishida (2021); Zinc-Induced Neurological Anti-Thrombosis and Thrombolysis in Children Under Severe Covid-19 Infective Pandemic Journal of Current Trends in Nursing \& Health Care 2:1-9.

9. Christopher J. Frederickson, Jae-Young Koh and Ashley Bush (2005); The neurobiolology of zinc in health and disease Nature Reviews Neuroscience 6: 449-462.

10. D. Neil Granger, Peter R. Kvietys (2015); Reperfusion injury and reactive oxygen species: The evolution of a concept. Redox Biology 6: 524-551.

11. Brittany E. Bannish1, Irina N. Chernysh, James P. Keener, Aaron L. Fogelson \& John W. Weisel (2017); Molecular and Physical Mechanisms of Fibrinolysis and Thrombolysis from Mathematical Modeling and Experiments. Science Reports 7: 1-14.
12. V. Murray, B. Norrving, P. A. G. Sandercock, A. Tere'nt, J. M. Wardlaw \& P. Wester (2010); The molecular basis of thrombolysis and its clinical application in stroke Journal Internal Medicine 267:191-208.

13. Yukinori Tamura (2021); The role of zinc homreostasis in the prevention of diabetes mellitus and cardiovascular diseases. Journal Atheroscler Thrombo 28: 1109-1122.

14. Susan Arentza, Jennifer Hunter, Guoyan Yanga, Joshua Goldenbergb, Jennifer Beardsley,et al (2020); Zinc for the prevention and treatment of SARS-CoV-2 and other acute viral respiratory infections: a rapid review Advances in Integrative Medicine 7: 252-260.

15. Sravya Kattula, James R. Byrnes, Alisa S. Wolberg (2017); Fibrinogen and Fibrin in Hemostasis and Thrombosis. Arterioscler Thromb Vasc Biol 37: e13-e21.

16. T. Ishida (2021); Zinc( II )-induced neurological anti-thrombus formation against severe COVID-19 infection Aditum Journal of Clinical and Biomedical Research 2: 1-8.

17. Kenan Aksu, Ayhan Donmez, and Gokhan Keser (2012); InflammationInduced Thrombosis: Mechanisms, Disease Associations and Management Current Pharmaceutical Design, 18: 1478-93.

18. Pavel Poredoš (2020); Inflammation and venous thromboembolic disease. Hematology \& Medical Oncology 5: 1-5.

19. James JM Loan, Caoimhe Kirby, Katherine Emelianova, Owen R Dando, Michael Tc Poon, et al (2021); Secondary injury and inflammation after intracerebral haemorrhage: a systematic review and meta-analysis of molecular markers in patient brain tissue. $\mathrm{J}$ Neurol Neurosurg Psychiatry 0: 1-7.

20. Ya-Wei Yan, Jun Fan, Shu-Ling Bai, Wei-Jian Hou, Xiang Li, et al (2016); Zinc Prevents Abdominal Aortic Aneurysm Formation by Induction of A20-Mediated Suppression of NF-KB Pathway PLOS One 11: e0148536.

21. Heinrich J. Audebert, Michaela M. Rott, Thomas Eck, Roman L. Haberl (2004); Systemic Inflammatory Response Depends on Initial Stroke Severity but Is Attenuated by Successful Thrombolysis. Stroke. 35: 2128-2133.

22. Sangyong CHOI, Xian LIU, Zui PAN (2018); Zinc deficiency and cellular oxidative stress: prognostic implications in cardiovascular diseases. Acta Pharmacologica Sinica 39: 1120-1132.

23. Masato Murata, Shuichi Hagiwara, Makoto Aoki, Jun Nakajima, Kiyohiro Oshima, et al (2019); The significance of the levels of fibrin/ fibrinogen degradation products for predicting trauma severity. Hong Kong Journal of Emergency Medicine 26: 143-150.

24. John C. Chapina, and Katherine A. Hajjara (2015); Fibrinolysis and the control of blood coagulation. Blood Rev. 29: 17-24.

25. Sara J. Henderson, Jing Xia, Huayin Wu, Alan R. Stafford, Beverly A. Leslie, et al (2016); Zinc promotes clot stability by accelerating clot formation and modifying fibrin structure. Thromb Haemost. 115: 53342.

26. S.J Henderson, A.R Stafford, B. A Leslie, P.Y Kim, Nima Vaezzadeh, et al (2015); Zinc delays clot lysis by attenuating plasminogen activation and plasmin-mediated fibrin degradation. Thromb Haemost. Jun 113:1278-1288.

27. Talia Knecht, Cesarb Borlongan, Ikebdela Peña (2018); Combination therapy for ischemic stroke: Novel approaches to lengthen therapeutic window of tissue plasminogen activator. Brain Circulation 4: 99-108.

28. Ralph Butkowski1, Derek King, Perla Cortes (2021); Zinc(II) Chelate Binds to Fibrinogen and Its aC Region. Advances in Biochemistry 9: 11-17. 
Citation: Ishida T (2021) Zinc( II ) Induced Neurological Thrombolytic Activities for COVID-19 Thrombus Prevention, Inflammation, Fibrin Degradation, Fibrinolysis of Dissolving Blood Clots and Blood Flow Reperfusion after Thrombolysis. Arch Surg Clin Case Rep 4: 158. DOI: 10.29011/2689-

29. Clemens Gutmann, Richard Siow, Adam M. Gwozdz, Prakash Saha, and Alberto Smith (2020); Reactive Oxygen Species in Venous Thrombosis. International Journal of Molecular Sciences. 21: 1918.

30. Min Li, Jing Zhou, Weifeng Jin, Xiaohong Li and Yuyan Zhang (2019); Danhong Injection Combined With t-PA Improves Thrombolytic Therapy in Focal Embolic Stroke. Frontiers in Pharmacology 9: 308.

31. Jing Xia, Li-Heng Caia, Huayin Wu, Frederick C. MacKintosh, and David A. Weitz (2021); Anomalous mechanics of Zn2+-modified fibrin networks PNAS 118 : e2020541118.

32. C. Michael Gibson, Sabina Murphy, Ian B. A. Menown, R F Sequeira, R Greene, et al (1999); Determinants of Coronary Blood Flow After Thrombolytic Administration. Journal of the American College of Cardiology 34: 1403-1412.

33. Sanjeev Kiran Gotru, Johanna P. vanGefen, Magdolna Nagy, Elmina Mammadova-Bach, et al (2019); Defective Zn2+ homeostasis in mouse and human platelets with $\alpha$ - and $\delta$-storage pool diseases Science Reports 9: 1-7.

34. Yoshiko Uesugi, Hiroyuki Kawata, Yoshihiko Saito, and Yasuhiko Tabata (2012); Ultrasound-responsive thrombus treatment with zinc-stabilized gelatin nano-complexes of tissue-type plasminogen activator Kyoto University Research Information Repository Kyoto Univ. 20: 224-34.

35. Jaime Emmetsberger, Martine M. Mirrione, Chun Zhou, Monica Fernandez-Monrea, Mustafa M. Siddiq, et al (2010); Tissue Plasminogen Activator Alters Intracellular Sequestration of Zinc through Interaction with the Transporter ZIP4. The Journal of Neuroscience. 30: 6538-6547.

36. Carmen Roncal, Sara Martinez de Lizarrondo, Agustina Salicio, Arnaud Chevilley, Jose A. Rodriguez, et al (2017); New thrombolytic strategy providing neuroprotection in experimental ischemic stroke: MMP10 alone or in combination with tissue-type plasminogen activator Cardiovascular Research 113: 1219-1229.

37. Talia Knecht, Cesar Borlongan, Ike dela Peña (2018); Combination therapy for ischemic stroke: Novel approaches to lengthen therapeutic window of tissue plasminogen activator. Brain Circulation. 4: 99-108.

38. Qing-Zhang Tuo, Zhen-Yu Liuyang, Peng Lei, Xiong Yan, Yang-Ping Shentu,et al (2018); Zinc induces CDK5 activation and neuronal death through CDK5-Tyr15 phosphorylation in ischemic stroke. Cell Death and Disease 9: 870 .

39. Jaspreet Kaur, Zonghang Zhao, Gary M. Klein, Eng H. Lo, and Alastair M. Buchan (2004); The Neurotoxicity of Tissue Plasminogen Activator. Journal of Cerebral Blood Flow \& Metabolism 24: 945-963.
40. Elmar Busch, Karsten Kruger, Peter R. Allegrini, Christian M. Kerskens, Michael L. Gyngell, et al(1998); Reperfusion After Thrombolytic Therapy of Embolic Stroke the Rat: Magnetic Resonance and Biochemical Imaging Journal of Cerebral Blood Flow and Metabolism 18: $407-418$

41. Mason Daniel Hurd, Isha Goel, Yasuyuki Sakai, Yuji Teramura (2021); Current status of ischemic stroke treatment: From thrombolysis to potential regenerative medicine. Regenerative Thrapy 18: 408-417.

42. Zihui Wang, Xinge Yu1, Yang V Li (2017); Zinc chelation promotes streptokinase-induced thrombolysis in vitro Int J Physiol Pathophysiol Pharmacol 9: 137-146.

43. Ming-Shuo Sun, Hang Jin, Xin Sun, Shuo Huang, Fu-Liang Zhang, et al (2018); Free Radical Damage in Ischemia-Reperfusion Injury: An Obstacle in Acute Ischemic Stroke after Revascularization Therapy. Oxidative Medicine and Cellular Longevity 2018: 3804979.

44. Xiang Wang, Yun-Feng Fu, Xiao Liu, Guo Feng, Dan Xiong, et al (2018); ROS Promote Ox-LDL-Induced Platelet Activation by UpRegulating Autophagy Through the Inhibition of the PI3K/AKT/ mTOR Pathway. Cell Physiol Biochem 50: 1779-1793.

45. Wolfgang Eberhardt, Karl-Friedrich Beck, and Josef Pfeilschifter (2002); Cytokine-induced expression of tPA is differentially modulated by NO and ROS in rat mesangial cells. Kidney International. 61: 20-30.

46. Carmen Domínguez, Pilar Delgado, Angel Vilches, Pilar MartínGalla'n, Marc Ribo', et al (2010); Oxidative Stress After ThrombolysisInduced Reperfusion in Human Stroke. Stroke. 41: 653-660.

47. Moinay Kim, Joonho Byun, Yeongu Chung, Si Un Lee, Ji Eun Park, et al (2021); Reactive Oxygen Species Scavenger in Acute Intracerebral Hemorrhage Patients. Stroke. 52: 1172-1181.

48. Matthew J. Flick (2021); Mechanism of ICH with tPA thrombolysis. Blood. 138: 8-9.

49. Ishida T (2020); Elucidation of Zinc-Induced Antiangiogenic Molecular Mechanism as "Therapy Treatment of Cancer and Tumor Disease". Clinical Research in Immunology 3: 1-7.

50. T. Ishida (2021); Zinc(II)-Induced Immunological Antiviral Activities for COVID-19 Prevention, Respiratory and Pulmonary Infection, AntiThrombus Formation, and ZAP-Mediated RNA Replication Journal of Immunology and Immunotherapy 4(1): 1-10. 